CASE REPORT

\author{
X. LV \\ Y. Li \\ M. Lv \\ A. Liu \\ J. Zhang \\ Z. Wu
}

\section{Trigeminocardiac Reflex in Embolization of Intracranial Dural Arteriovenous Fistula}

SUMMARY: We report a case of immediate reproducible and reflexive response of asystole upon stimulation of Onyx injection during embolization of a tentorial dural arteriovenous fistula in a 53-yearold man. Upon recognition of the reflexive relationship between Onyx injection and increased vagal tone, the patient was given anticholinergic in an effort to block cholinergic hyperactivity. After atropine was given, no further dysrhythmias occurred.
$\mathbf{P}$ revious articles have described trigeminocardiac reflex (TCR) during intracranial operations, ophthalmic surgery, and microcompression of the trigemimal ganglion. ${ }^{1-4}$ We report a case of likely TCR seen upon mechanical stimulation of the middle meningeal artery in the spinosum foramen during embolization of a tentorial dural arteriovenous fistula (DAVF). This is the first case of TCR during transarterial embolization of DAVF.

\section{Case Report}

A 53-year-old man presented with subarachnoid hemorrhage 2 years previously, and although conservative treatment was given at another hospital, headaches were still persistent. Cerebral angiograms were obtained and showed a DAVF fed by the right internal maxillary artery, the dorsal meningeal artery from the right meningohypophyseal trunk, and branches of the right posterior cerebral arteries. Venous outflow emptied into the right sigmoid sinus via the right dilated cerebellar cortical veins. Electrocardiogram (ECG) was normal. Recommendation was made for transarterial embolization of this lesion.

Endovascular treatment was performed with the patient under standard anesthetic protocol. Anesthesia was induced with fentanyl (3 $\mu \mathrm{g} / \mathrm{kg})$, followed by vecurium (1 mg/kg) and propofol $(2 \mathrm{mg} / \mathrm{kg}$ ). After the intubation of the trachea, the lungs were mechanically ventilated with a mixture of air and oxygen $\left(\mathrm{FIO}_{2}=0.5\right)$. Anesthesia was maintained with renifentanyl $(0.02 \mu \mathrm{g} / \mathrm{kg} / \mathrm{min})$, and additional boluses of propofol $(7 \mathrm{mg} / \mathrm{kg} / \mathrm{h})$ and vecurium $(3 \mathrm{mg} / \mathrm{h})$ were administered. A bilateral $6 \mathrm{~F}$ femoral access was prepared, and the tip of a $6 \mathrm{~F}$ guiding catheter was placed inside the right external carotid artery, while a $5 \mathrm{~F}$ diagnostic catheter with continuous heparinized flush was positioned in the right vertebral artery for selective control angiograms. Using a road-mapping technique and fluoroscopic guidance, a Marathon microcatheter (ev3, Irvine, Calif.) was advanced over a 0.008 -inch Mirage guidewire (ev3) in the right middle meningeal artery up to the fistula zone. Under biplane road-mapping, the catheter was slowly flushed with $0.25 \mathrm{~mL}$ of dimethyl-sulfoxide (DMSO) over 40 seconds, and this was followed by injection of Onyx 18 (ev3).

The embolic material achieved antegrade filling of the malformation, but then formed a small reflux of several millimeters in length over the tip of the microcatheter. After waiting one minute for the

Received February 6, 2007; accepted after revision March 1

From the Beijing Neurosurgical Institute and the Neurointerventional Department, Beijing Tiantan Hospital, Capital Medical University, Beijing, China.

Please address correspondence to Wu Zhongxue, MD, Beijing Neurosurgical Institute, Neuroradiological Department, No. 6, Tiantan Xili, Beijing, China 100050; e-mail: ttyyzjb@sina.com

DOI 10.3174/ajnr.A0675 reflux to solidify, the injection was continued gently. Again, some antegrade filling was achieved, but shortly, another short reflux was noted. This cycle was repeated until a plug of the embolic agent was obtained so that the material would penetrate distally. In the meantime, caution was exerted to avoid inadvertent embolization of posterior circulation during the slow injection of Onyx. Patency of the vertebral artery was checked frequently during the intermittent injection of the embolic material. However, when the Onyx plug reached the foramen spinosum, an asystole was noted following Onyx injection. We stopped the injection, and the heart rate returned to the baseline. When another injection was performed, the arterial rhythm decreased again. After 2 doses of atropine $0.5 \mathrm{mg}$ were intravenously administered at 11:45 and 12:00, the reflex did not recur during the remainder of the procedure. The amount of injected Onyx was 2.5 $\mathrm{mL}$, and duration time of injection was 80 minutes. There was no difficultly in retrieving the catheter. After embolization, control angiograms showed immediate occlusion of the feeders from the external carotid artery and persistent injection from the right internal carotid artery and the right posterior cerebral artery. The patient had no events after the procedure. The residual lesion would be handled at the second session.

\section{Discussion}

The phenomenon of bradycardia during Onyx injection has not previously been described. During Onyx injection, bradycardia was reported by the anesthesiologist and resolved upon cessation of injection as the Onyx refluxed to the foramen spinosum. The patient's vital signs returned to baseline. Three subsequent individual and temporally discrete injections reproduced similar responses of bradycardia. After intravenous administration of atropine, the response was no longer reproducible, and the procedure could be completed. The reflex bradycardia seen during Onyx injection is not likely attributable to other factors. The entire procedure was performed under a standard anesthetic protocol. We did not stimulate the brain to produce the reflex. Research of anesthesia monitoring in patients treated with Onyx embolization for intracranial aneurysms showed no changes in heart rate or blood pressure following DMSO and Onyx injections, nor were any arrythmias observed. ${ }^{5}$ We consider that this response was a TCR, which has been previously described in the literature as a reflexive response of bradycardia, hypotension, and gastric hypermobility seen upon mechanical stimulation in the distribution of the trigeminal nerve. ${ }^{1}$ Clinically, TCR has been reported to occur during craniofacial surgery, ${ }^{2}$ tumor resection in the cerebellopontine angle ${ }^{3}$ and falx cerebri, ${ }^{1}$ and trans- 
sphenoidal surgery for pituitary adenomas. ${ }^{4}$ Review of the literature failed to identify a previously reported case of TCR during embolization of a DAVF. TCR as a physiologic reflex has been clarified. ${ }^{6}$ The reflex was reproducible during light manipulation of the pedicle of the middle meningeal artery, so the reflex probably began from the foramen spinosum. We feel that the anatomic studies of Penfield and McNaughton ${ }^{7}$ reasonably display the nervous innervation of the dura and a rational pathway from the dura to the vagal motor nucleus. ${ }^{1}$ They described a mandibular branch of trigeminal nerve, the nervus spinosus, that follows the middle meningeal artery outside the cranium and through the foramen spinosum to innervate the middle fossa dura. We think that direct compression of the nervus spinosus by the dilated middle meningeal artery due to formation of Onyx plug produces and sends neuronal signals via the Gassarian ganglion to the sensory nucleus of the trigeminal nerve, forming the afferent pathway of the reflex arc. This afferent pathway continues along the short internuncial nerve fibers in the reticular formatio to connect with the efferent pathway in the motor nucleus of the vagus nerve and causes bradycardia. When stimulation of the foramen spinosum causes TCR, treatment of hemodynamic instability consists of ceasing procedure and administering anticholinergic drugs. Atropine was shown to effectively extinguish the TCR in our patient. Anticholinergic drugs are not given prophylactically because they can cause refractory arrhythmias. ${ }^{1}$

\section{Conclusion}

The present case gives evidence for the first time that TCR may occur during transarterial embolization of DAVF with Onyx, leading to a significant decrease in heart rate under a standard anesthetic protocol. This case confirmed that the reflex was blunted by the anticholinergic effects of atropine. Recognition of TCR allows for early detection and appropriate treatment.

\section{References}

1. David F, Bawer BA, Youkilis A, et al. The falcine trigeminocardiac reflex: case report and review of the literature. Surg Neurol 2005;63:143-48

2. Panneton WM, Yavari P. A medullary dorsal horn relay for the cardiorespiratory response evoked by stimulation of the nasal mucosa in the muskrat Ondatra zibethicus: evidence for excitatory amino acid transmission. Brain Res 1995;691:37-45

3. Schaller B, Probster R, Strebal S, et al. Trigemino-cardiac reflex during surgery in the cerebellopontine angle. J Neurosurg 1999;90:215-20

4. Schaller B. Trigemino-cardiac reflex during transsphenoidal surgery for pituitary adenomas. Clin Neurol and Neurosurg 2005;107:468-74

5. Pamuk AG, Sactci I, Cekirge HS, et al. A contribution to the controversy over dimethyl sulfoxide toxicity: anesthesia monitoring results in patients treated with Onyx embolization for intracranial aneurysms. Neuroradiology 2005; $47: 380-86$

6. Schaller B. Trigeminocardiac reflex: A clinical phenomenon or a new physiological entity? J Neurol 2004;251:658-65

7. Penfield W, McNaughton F. Dural headache and innervation of the dura matter. Arch Neurol Psychiatr 1940;44:43-75 